|| ISSN(online): 2589-8698 || ISSN(print): 2589-868X ||

International Journal of Medical and Biomedical Studies

Available Online at www.ijmbs.info

NLM (National Library of Medicine ID: 101738825)

Index Copernicus Value 2019: 79.34

Original Research Article

Volume 5, Issue 8; August: 2021; Page No. 287-289

\title{
CLINICAL PROFILE OF NEONATES WITH RESPIRATORY DISTRESS
}

\section{Dr. Sandeep Kulhari, Dr Deepak Choudhary}

${ }^{1,2}$ MBBS, MD(Paeditrics)

Article Info: Received 06 July 2021; Accepted 07 August 2021

DOI: https://doi.org/10.32553/ijmbs.v5i8.2136

Corresponding author: Dr Deepak Choudhary

Conflict of interest: No conflict of interest.

\begin{abstract}
Background: Respiratory distress (RD) is a challenging problem and is one of the most common causes of admission in neonatal intensive care unit (NICU).

Methods: A prospective study was conducted on 50 cases. Term, pre-term and post-term babies both in-borns and out-borns cases were included in the study.

Results: Of the 50 cases admitted with RD, 31 babies (62\%) were delivered vaginally and 19 (38\%) by lower segment caesarean section (LSCS). There were $33(66 \%)$ males and 17 (34\%) females in the study. There were $23(46 \%)$ pre-term babies, $26(52 \%)$ term and $1(2 \%)$ post-term neonates who were admitted with RD. The majority of cases $42(82 \%)$ presented with increased respiratory rate, chest in drawings. 41 (82\%) babies had flaring of alae nasi.

Conclusion: Increased respiratory rate along with chest in drawing, flaring of alae nasi are the presentation of RD in majority of cases.
\end{abstract}

Keywords: Respiratory distress (RD), Flaring of alae nasi are, Tachypnea.

\section{Introduction}

Respiratory distress (RD) is a challenging problem and is one of the most common causes of admission in neonatal intensive care unit (NICU). ${ }^{1}$ The neonatal mortality rate varies by state but, overall, it is reported to be 39 a 1000 live births in India. ${ }^{2}$ Neonatal period is a very vulnerable period of life due to many problems which can occur. Most of the causes of neonatal morbidity and mortality are preventable. $^{3}$ The common causes of $\mathrm{RD}$ in neonates includes transient tachypnea of the newborn (TTN), hyaline membrane disease (HMD), birth asphyxia, pneumonia, meconium aspiration syndrome (MAS), and other miscellaneous causes. ${ }^{4,5}$

The severity of respiratory distress can be assessed by Downe's scoring system which includes parameters such as respiratory rate, cyanosis, retractions, grunting and air entry in both the lungs. 2 Common causes of respiratory distress are respiratory distress syndrome, transient tachypnea, pneumonia, aspiration syndromes, pneumothorax and air leaks, pulmonary edema, pleural effusion and pulmonary hemorrhage. ${ }^{1}$

Since the millennium development goals (MDG) were formed, progress toward reducing child mortality has accelerated but remains insufficient to achieve MDG. In particular, global progress toward reducing neonatal deaths that is deaths during the first 28 days of life has been slow and neonatal deaths now account for a greater proportion of child deaths than in 1990 . India accounts for $27.3 \%$ of total neonatal deaths in the world. Distress NRD is ranging from $2.2 \%$ to $7.6 \%$ in developed countries and from $0.7 \%$ to
$8.3 \%$ in India. ${ }^{6}$ It is caused by the delay in the absorption of fluid in the lungs after birth (i.e. excessive lung fluid).

\section{Materials and Methods}

Study Design - A prospective study was conducted on who were admitted in NICU.

Inclusion Criteria -Both in-born and out-born neonate admitted to NICU with RD.

Exclusion Criteria -

- $\quad$ Babies more than 28 days

- Babies <28 weeks of age.

\section{Data Collection}

Neonates were classified as term, pre- and post-term were enrolled as cases with RD on the basis of clinical profile.

A detailed proforma including name, age, sex, and residence was obtained. Neonatal data recorded includes weight of the baby, gestational age, mode of delivery, APGAR score, if available, the need for resuscitation after birth, onset of RD and resolution of RD.

Factors related to labor and deliveries were assessed including type of delivery normal vaginal or $\mathrm{C}$-section. Elective or emergency, place of delivery, (any associated complications like; prolonged rupture of the membrane more than $24 \mathrm{~h}$, prolonged labor, meconium stained liquor, antepartum hemorrhage and others).

Maternal information was recorded including age, parity and any systemic diseases. Other risk factors include 
delivery prior to 38 weeks of gestation, male sex, low birth weight and macrosomia and maternal diseases such gestational diabetes and asthma. The cases were diagnosed clinically by the presence of at least 2 of the following criteria, namely RR of $60 / \mathrm{min}$ or more, subcostal in the drawing, and retraction, suprasternal in drawing, flaring of alae nasi, expiratory grunt and cyanosis. The diagnosis of clinical conditions producing $\mathrm{RD}$ was based mainly on careful scrutiny of the history, clinical and radiological findings. Continuous monitoring of oxygen saturation was done using pulse oxymeter. The arterial blood gas (ABG) analysis was done frequently in unstable babies and with changes in ventilator settings. Blood glucose was monitored regularly using the dextrostix, sepsis workup was done when clinically indicated, endotracheal tube and blood culture sensitivity were ordered if septicemia or pneumonia was suspected as per guidelines baby was mechanically ventilated, and modified the settings according to $A B G$ analysis.

\section{Results}

Of the $102(34 \%)$ cases admitted with RD, 61 babies $(60 \%)$ were delivered vaginally and $41(40 \%)$ by lower segment caesarean section (LSCS)

Table 1: General information

\begin{tabular}{|l|l|l|l|}
\hline Variable & No of babies & Percentage \\
\hline \multirow{2}{*}{ Type of delivery } & Normal vaginal delivery & 31 & $62.00 \%$ \\
\cline { 2 - 4 } & LSCS & 19 & $38.00 \%$ \\
\hline \multirow{3}{*}{ Gender } & Male & 33 & $66.00 \%$ \\
\cline { 2 - 4 } & Female & 17 & $34.00 \%$ \\
\hline \multirow{3}{*}{ Gestational age } & Term & 26 & $52.00 \%$ \\
\cline { 2 - 4 } & Pre term & 23 & $46.00 \%$ \\
\cline { 2 - 4 } & Post term & 1 & $2.00 \%$ \\
\hline
\end{tabular}

Of the 50 cases admitted with RD, 31 babies (62\%) were delivered vaginally and 19 (38\%) by lower segment caesarean section (LSCS). There were $33(66 \%)$ males and $17(34 \%)$ females in the study. There were $23(46 \%)$ pre-term babies, 26 $(52 \%)$ term and $1(2 \%)$ post-term neonates who were admitted with RD.

Table 2: Clinical profile

\begin{tabular}{|l|l|l|}
\hline Clinical profile & No of babies & Percentage \\
\hline Tachypnea & 42 & $84.00 \%$ \\
\hline Falring of alae nasi & 41 & $82.00 \%$ \\
\hline Chest in drawing & 42 & $84.00 \%$ \\
\hline Grunting & 21 & $42.00 \%$ \\
\hline Cyanosis & 20 & $40.00 \%$ \\
\hline
\end{tabular}

The majority of cases $42(82 \%)$ presented with increased respiratory rate, chest in drawings. 41 (82\%) babies had flaring of alae nasi.

\section{Discussion}

Of the 50 cases admitted with RD, 31 babies (62\%) were delivered vaginally and $19(38 \%)$ by lower segment caesarean section (LSCS). There were $33(66 \%)$ males and $17(34 \%)$ females in the study. There were 23 (46\%) preterm babies, $26(52 \%)$ term and $1(2 \%)$ post-term neonates who were admitted with RD.

A study done Santhosh et al. in their study showed $39 \%$ term and $61 \%$ pre-term neonates who were developed RD in newborn. The incidence of MAS in developed countries is on the decline possibly due to improved obstetric care ${ }^{7}$.

Pneumothorax usually develops secondary to an underlying disease process but can occur spontaneously in $1 \%$ of newborns around the perinatal period, although only about $10 \%$ of these are symptomatic. ${ }^{8}$ The majority of cases 42 $(82 \%)$ presented with increased respiratory rate, chest in drawings. $41(82 \%)$ babies had flaring of alae nasi. TTN babies develop an oxygen requirement that necessitates admission to the neonatal unit for a few days accounting for approximately $10 \%$ of all newborn term admissions. ${ }^{9}$ In a review of TTN, Yurdakok suggests a genetic link between TTN and later onset asthma. ${ }^{10}$ A similar study done by Keerti et al. showed that of all the symptoms, grunting, flaring of alae nasi had high specificity for RD in newborn while tachypnea, chest retractions and difficulty in feeding has high sensitivity. ${ }^{11}$ Every year a significant number of termborn infants are admitted to neonatal units for management of their respiratory distress. ${ }^{12}$ And that the most common clinical presentations were tachypnea, flaring of alae nasi and chest retraction. It was also observed that there was a male preponderance with a ratio more than 1.5:1. ${ }^{1}$ And showed inversely proportional to gestational age and birth weight, and $\mathrm{C}$ section can favor the onset of RD in newborns. ${ }^{1}$

\section{Conclusion}

Increased respiratory rate along with chest in drawing, flaring of alae nasi are the presentation of RD in majority of cases. 


\section{References}

1. Swarnakar K, Swarnakar M. Neonatal respiratory distress in early neonatal period and its outcome. Int J Biomed Adv Res 2015;6:643-7.

2. Niswade A, Zodpey SP, Ughade S, Bangdiwala SI. Neonatal morbidity and mortality in tribal and rural communities in central India. Indian J Community Med 2011;36:150-8.

3. Gauchan E, Basnet S, Koirala DP, Rao KS. Clinical profile and outcome of babies admitted to Neonatal Intensive Care Unit (NICU). J Inst Med 2011;33:15 .

4. Shah GS, Yadav S, Thapa A, Shah L. Clinical profile and outcome of neonates admitted to Neonatal Intensive Care Unit (NICU) at a tertiary care centre in Eastern Nepal. J Nepal Paediatr Soc 2013;33:177-81.

5. Edwards MO, Kotecha SJ, Kotecha S. Respiratory distress of the term newborn infant. Paediatr Respir Rev 2013;14:29-36.

6. Avery ME, Gatewood OB, Brumley G. Transient tachypnea of newborn. Possible delayed resorption of fluid at birth. Am J Dis Child 1966;111:380-5.
7. Greenough A, Morley C, Roberton N. Acute respiratory diseases in the newborn. In: Roberton $\mathrm{N}$, editor. Textbook of Neonatology. 2nd ed. London: Churchill Livingstone; 1992. p. 385-504.

8. Santhosh S, Kumar K, Adarsha E. A clinical study of respiratory distress in newborn and its outcome. Indian J Neonatal Med Res 2013;1:2-4.

9. Tutdibi E, Gries K, Bücheler M, Misselwitz B, Schlosser RL, Gortner L. Impact of labor on outcomes in transient tachypnea of the newborn: Population-based study. Pediatrics 2010;125:e57783.

10. Yurdakök M. Transient tachypnea of the newborn: What is new? J Matern Fetal Neonatal Med 2010;23 Suppl 3:24-6.

11. Consortium on Safe Labor, Hibbard JU, Wilkins I, Sun L, Gregory K, Haberman S, et al. Respiratory morbidity in late preterm births. JAMA 2010;304:419-25.

12. Tutdibi E, Gries K, Bücheler M, Misselwitz B, Schlosser RL, Gortner L. Impact of labor on outcomes in transient tachypnea of the newborn: Population-based study. Pediatrics 2010;125:e57783. 